\title{
Recent Developments in Basic and Clinical Hematopoietic Stem Cell Transplantation
}

\author{
Shinpei Kasakura \\ Kobe City General Hospital, \\ Kobe, Japan
}

Int J Hematol. 2003;77:1-2.

(C)2003 The Japanese Society of Hematology

\begin{abstract}
Allogeneic hematopoietic stem cell transplantation (HSCT) is an effective therapy for patients with hematological malignancies, including various types of leukemia, lymphomas, and multiple myeloma. It is also the most effective therapy for severe aplastic anemia in young patients. Highdose chemotherapy followed by autologous HSCT has been similarly effective in the treatment of hematological malignancies and even some solid tumors.
\end{abstract}

In the 1950s and 1960s, many patients with leukemia or aplastic anemia underwent bone marrow transplantations. However, these transplantations were generally unsuccessful, except in the patients who had identical-twin donors. Early failures were primarily due to a lack of knowledge of human histocompatibility antigens. The modern era of bone marrow transplantation began in the late 1960s with a growing knowledge of the HLA system and the development of techniques of histocompatibility typing. Initially bone marrow only from HLA-identical siblings was used for transplantation; later marrow from HLA-mismatched related donors was used, and now even marrow from HLA-matched unrelated volunteer donors is used. Furthermore, not only marrow but also hematopoietic stem cells derived from peripheral blood or umbilical cord blood are now used for transplantation. These additional cell sources have led to increasing numbers of transplantations. Over the past 3 decades, HSCT has been performed with increasing success.

Dr. E.D. Thomas won a Nobel prize in 1990 for his great contributions to the development of clinical bone marrow transplantation. As a former student of Dr. Thomas, I am honored to introduce this issue of the International Journal of Hematology with a minireview of recent developments in basic and clinical HSCT that highlights each author's contribution.

Over the past few years nonmyeloablative allogeneic HSCT "minitransplantation" has been developed as an important alternative to conventional myeloablative allogeneic HSCT for treating hematological malignancies in older or medically unfit patients who cannot tolerate myeloablative conditioning. Until recently, it was believed that complete ablation of the host hematopoietic microenvironment was a prerequisite to achievement of engraftment of allogeneic marrow. However, recent studies of engraftment in nonmyeloablated hosts have challenged this concept. It is now clear that creation of marrow space by intensive chemoradiation therapy is not obligatory for engraftment to occur. It has also been shown that mixed chimerism, which can be established with nonmyeloablative conditioning, induces a relative resistance to graft-versus-host disease (GVHD). Delayed infusion of donor lymphocytes (DLI) after nonmyeloablative allogeneic HSCT can increase donor chimerism in recipients and is an effective approach to preventing relapse of hematological malignancies after HSCT. The concept of minitransplantation is based on these findings. Rainer Storb is one of the pioneers in the development of minitransplantation. He successfully designed a nonmyeloablative conditioning regimen for clinical use, consisting of low-dose total body irradiation (TBI) before HSCT and mycophenolate mofetil (MMF) combined with cyclosporine after HSCT. This regimen provided sufficient immunosuppression for achievement of engraftment of allogeneic HSC and allowed for the subsequent development of a graft-versus-malignancy $(\mathrm{GVM})$ effect. In this issue of the Journal, Georges and Storb [1] review the biological background, current clinical applications, and indications for this novel treatment approach. They also review the updated results of an ongoing multicenter study investigating the safety and efficacy of nonmyeloablative allogeneic HSCT using a 2-Gy TBI-based regimen in patients with advanced hematological malignancies who are ineligible for conventional myeloablative conditioning. In addition, Georges and Storb review the results of nonmyeloablative HSCT trials from other transplantation centers.

GVHD continues to be a problem in allogeneic HSCT. Our view of GVHD has evolved significantly over the last few years. The basic pathophysiology of GVHD is much more complex than previously appreciated. A better understanding of the pathophysiology of GVHD has led to the development of many new ways of preventing and treating GVHD. We now know that acute GVHD is caused by a combination of host tissue damage resulting from the conditioning regimen, direct cytotoxic damage of host target cells by donor cytotoxic T-lymphocytes (CTL), and effects of 
cytokines, endotoxin, and NO. T-cell-depletion of transplants prevents GVHD, a result indicating that the donor T-cell is critical for development of GVHD. Several studies have provided evidence that clarifies the role of the Fas/Fas $\mathrm{L}$ pathway and tumor necrosis factor $\alpha(\mathrm{TNF}-\alpha)$ in specific acute GVHD target-organ damage. The Fas/Fas L pathway is associated with hepatic and cutaneous GVHD and TNF- $\alpha$ with intestinal and cutaneous GVHD. The cytokine cascade is believed to cause the clinical manifestations of GVHD. However, details of the interaction between cytokines and the exact role of each cytokine in this cascade leading to GVHD have not been completely elucidated. Recent studies suggest that acute GVHD is characterized by the overproduction of T-helper (Th)1 cytokines (interleukin [IL]-2, interferon $\gamma$ ), whereas chronic GVHD is associated with the overproduction of Th2 cytokines (IL-4, IL-5, IL-6, IL-10, IL-13). Th1/Th2 imbalance seems to play a critical role in the induction of acute versus chronic GVHD. It is well known that GVHD is associated with decreased relapse of hematological malignancies after allogeneic HSCT. This finding strongly suggests that allogeneic HSCT induces GVHD as well as an immune-mediated GVM effect. So far, many attempts have failed to separate a GVM effect from GVHD. The goal is to develop an approach that would prevent GVHD and, at the same time, maintain a GVM effect. In this issue of the Journal, Joachim Deeg [2] introduces the current view of the pathophysiology of GVHD and discusses its implications for designing approaches to prevent and treat GVHD.

Relapse of the underlying malignancy continues to be one of the major problems after both autologous and allogeneic HSCT. An immune-mediated GVM effect can prevent relapse after allogeneic HSCT for hematological malignancies. The observation that depletion of T-lymphocytes in marrow grafts reduces incidence of GVHD but increases relapse rate suggests that both the GVM effect and GVHD are mediated by donor T-lymphocytes. Many investigators have tried to develop an approach that reduces GVHD while maintaining the GVM effect. DLI after allogeneic HSCT has been shown to induce remission in patients with hematological malignancies. Furthermore, compared with donor lymphocyte administration at the time of transplantation, DLI administration is associated with a reduced incidence of GVHD. These data suggest that DLI after allogeneic HSCT may achieve the goal of reducing GVHD while maintaining the GVM effect. Recent progress in characterizing tumor-specific antigens has provided new tools in developing cancer vaccines. Antitumor immunity has been successfully induced by administration of autologous dendritic cells loaded with relevant tumor-specific peptides. Tumor-specific peptides may be delivered with cytokines such as IL-2, IL-12, or granulocyte-macrophage colonystimulating factor and costimulatory molecules such as B7 to boost tumor-specific immune responses. Treatment using these new immunotherapies may be tried in posttransplantation patients. In this issue, Sally Arai and Hans Klingemann [3] extensively review various immunotherapeutic approaches to prevent and treat relapse occurring after both autologous and allogeneic HSCT.

It has been demonstrated that virtually all cell types found in adult organs can be generated from embryonic stem cells. The technique of transplanting cells derived from embryonic stem cells has opened up many clinical possibilities for the replacement of tissue cells lost because of damage caused by diseases or injuries. More recently, it has been demonstrated that some tissues can be regenerated through the transplantation of placental/umbilical cord blood or even adult hematopoietic stem cells. Stem cells are now at the center of interest at all levels of research, to clinicians, and to society in general. Therefore, it is important to gain knowledge of the genes controlling stem cell function.

In this issue of the Journal, Gary Van Zant [4] reviews what is known about the genes controlling hematopoietic stem cell function. The ability to identify and ultimately manipulate the genes that regulate stem cell number, replication rate, and self-renewal capacity may have important clinical benefits. Van Zant discusses evidence suggesting that the characterization of some of these stem cell genes will shed light on mechanisms important in the aging process. $\mathrm{He}$ advances the hypotheses that stem cells accumulate cellular damage during aging and that the impaired function of stem cells in hematopoietic and in other self-renewing tissues limits longevity in animals and perhaps humans.

\section{References}

1. Georges G, Storb R. Review of "minitransplantation": nonmyeloablative allogeneic hematopoietic stem cell transplantation. Int $J$ Hematol. 2003;77:3-14.

2. Deeg HJ. New strategies for prevention and treatment of graft-versus-host disease and for induction of graft-versus-leukemia effects. Int J Hematol. 2003;77:15-21.

3. Arai S, Klingemann HG. Role of immunotherapy in stem cell transplantation. Int J Hematol. 2003;77:22-28.

4. Van Zant G. Genetic control of stem cells: implications for aging. Int J Hematol. 2003;77:29-36. 